\title{
EL SINSENTIDO DE HABLAR DE LITERATURA GRIS EN LA ÉPOCA 2.0
}

\author{
Francisco-Javier Martínez-Méndez y Rosana López-Carreño
}
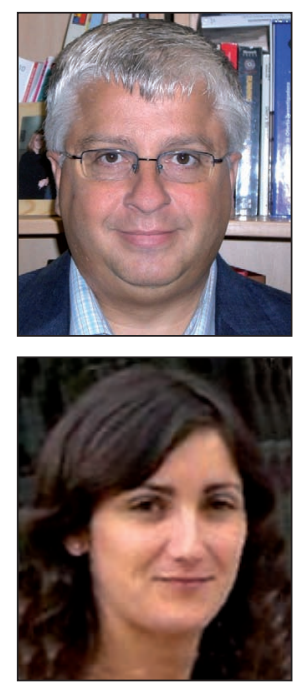

Francisco-Javier Martínez-Méndez es doctor en documentación por la Universidad de Murcia, profesor de tecnologías de la información en la Facultad de Comunicación y Documentación y principalmente desempeña su trabajo en el campo de la recuperación de información en la web.

Universidad de Murcia

Depto. de Información y Documentación Campus de Espinardo. 30100 Espinardo, Murcia http://irsweb.es javima@um.es

Rosana López-Carreño es doctora en documentación por la Universidad de Murcia, profesora de fuentes de información en la Facultad de Comunicación y Documentación y principalmente desempeña su trabajo en este campo y en el de los recursos de información y documentación educativa.

Universidad de Murcia Depto. de Información y Documentación Campus de Espinardo. 30100 Espinardo, Murcia rosanalc@um.es

\section{Resumen}

El vertiginoso avance que las TIC han introducido en nuestro campo no está siempre acompañado de actualizaciones de las bases teóricas, que corren peligro de convertirse en obsoletas e inservibles. Un ejemplo de este problema es el concepto "literatura gris", que prácticamente permanece inalterable en los textos y manuales de estudio en castellano. Esto no ha sido así a nivel internacional, donde han tenido lugar distintas conferencias para actualizar esta disciplina. Si bien el intento fue digno de mención, en el contexto actual de la web 2.0 el concepto de literatura gris no parece justificarse. Los clásicos hechos diferenciadores esgrimidos por los defensores de esta idea y muchos de aquellos que se han añadido a lo largo del tiempo, han ido quedando obsoletos. Hoy en día no tiene sentido hablar de literatura gris.

\section{Palabras clave}

Literatura gris, Internet, Web, Conceptualizaciones, Análisis teórico.

\section{The idea of grey literature makes no sense in the $\mathbf{2 . 0}$ era}

\begin{abstract}
The rapid progress that ICTs have introduced to our field is not always accompanied by updates of the theoretical bases that sometimes are in danger of becoming obsolete and useless. An example of this problem is the concept of "grey literature", which remains practically unchanged in the textbooks and study manuals written in Spanish. This has not been the case at an international level, where various international conferences have been organized to update this discipline. While the attempt was noteworthy, in the current context of web 2.0 the concept does not seem justified. The traditional differentiating features used by the proponents of this idea, and many of those that have been added over time, have become obsolete. Today it makes no sense to hold on to grey literature.
\end{abstract}

\section{Keywords}

Grey literature, Internet, Web, Conceptualization, Theoretical analysis.

Martínez-Méndez, Francisco Javier; López-Carreño, Rosana. "El sinsentido de hablar de literatura gris en la época 2.0". El profesional de la información, 2011, noviembre-diciembre, v. 20, n. 6, pp. 621-626. 


\section{Concepción obsoleta (clásica) de la literatura gris}

Si repasamos la literatura científica de los últimos años, no resulta raro encontrar situaciones donde la comunidad de autores se preocupa mucho más de presentar nuevos avances o nuevos contextos (como puede ser el caso que nos ocupa de la web 2.0), que de revisar conceptos (adaptando definiciones o bien directamente suprimiéndolos). De esta manera se produce la paradoja de disponer de unas aportaciones científicas actualizadas sobre un corpus de conocimiento que poco a poco va quedando obsoleto. Discutir el por qué de este fenómeno, al que ninguno de nosotros somos ajenos, puede llevarnos mucho tiempo y no es el objeto principal de este trabajo, pero creemos que se encuentra íntimamente ligado, al menos en nuestro campo, a la permanente discusión sobre qué es más importante: la ciencia o la tecnología.

En los años 40 surge el concepto de literatura gris (si bien no existe unanimidad en cuanto a la fecha, está claro que es cuando cobra mayor importancia dentro de la comunidad bibliotecaria), para referirse, en primer término, a los informes de carácter técnico o científico cuyos canales de difusión distaban mucho de ser los habituales del mundo editorial (De-Torres, 1998). Para Currás (1998) la literatura gris "puede obtenerse públicamente, no siendo convencional en su contenido, no estando bien controlada su publicación, ni siendo accesible por los canales normales de distribución lo que la hace difícil de obtener y de localizar. Además de ello son documentos de tipo muy variado, que van desde las publicaciones no revisadas hasta los documentos de contenido no muy concreto".

Este enfoque clásico de la literatura gris es el que predomina en los textos científicos, especialmente los publicados en castellano (Pujol, 1995; Martín, 1995; Cordón, 1999; Carrizo, 2000) y, por tanto, es el más utilizado a la hora de definir y caracterizar a este conjunto de documentos. Los textos docentes corren peligro de quedarse obsoletos y desfasados, reincidiendo en la idea del "conjunto heterogéneo de documentos de difícil acceso, impresos, producidos y difundidos por cauces distintos a las monografías y artículos". Se obvian, por un lado, los esfuerzos realizados por muchos autores e investigadores para la actualización y renovación de esta disciplina (algo imperdonable porque se supone que los docentes e investigadores deberían actualizar sus fuentes) y, por otro lado, la evolución que se ha producido en el mundo de la comunicación científica hasta llegar al entorno actual de la web 2.0 donde la popularización de servicios de autoedición y coedición electrónica de documentos (blogs, wikis, webquests ${ }^{1}$, etc.) y la profusión de repositorios documentales en acceso abierto implementados según las especificaciones del protocolo OAI-PMH, hacen que esta percepción clásica quede, al menos, algo desajustada, pudiendo llegarse a afirmar que totalmente desbordada e inservible. Más si cabe en el contexto actual, basado en el paradigma read/write de la web 2.0 propuesto por Daugherty, quien, en una tormenta de ideas online llegó a sugerir un "renacimiento de la web", contexto donde cabría preguntarse si existe de verdad algún tipo documental que escapase a la definición clásica, de manera que la cuestión que verdaderamente tendría sentido sería: "de continuar

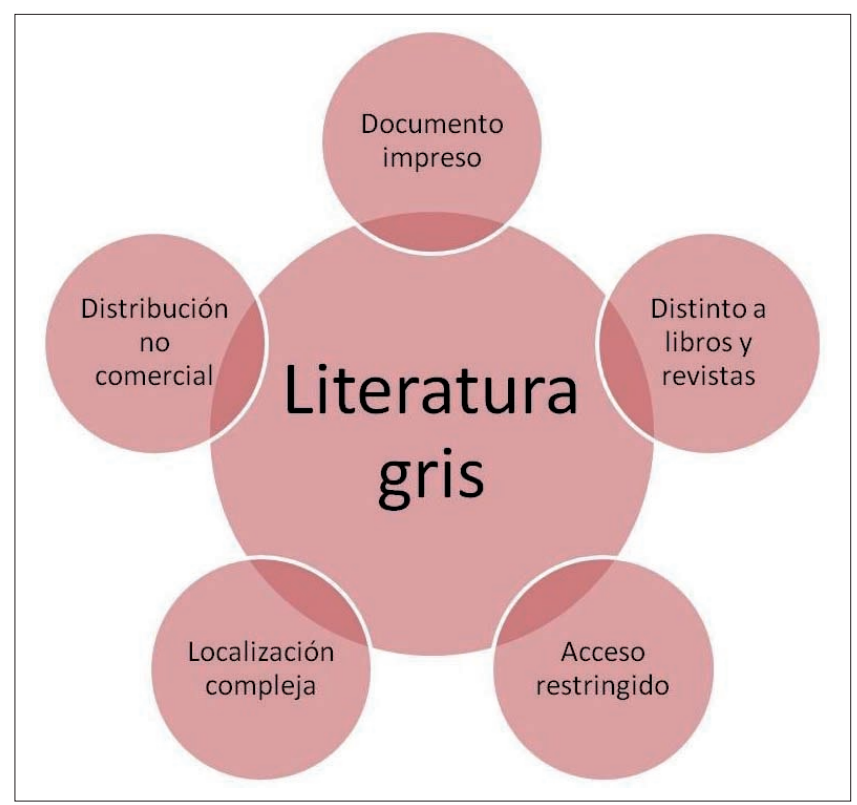

Figura 1. Características tradicionales de la literatura gris

vigente esta idea, ¿sigue existiendo el negro sobre blanco o todo es gris?"

\section{Evolución del concepto de literatura gris: ¿ahora todo vale?}

En un esfuerzo de actualización del concepto, la 12 $\stackrel{a}{ }$ Conferencia internacional sobre literatura gris (Praga, 2010), lo define "como sinónimo de múltiples tipos de documentos producidos en los ámbitos gubernamentales, académicos, empresariales e industriales tanto en formato impreso como electrónico, protegidos por derechos de propiedad intelectual y que son de suficiente calidad para ser recogidos y conservados en los fondos de las bibliotecas o repositorios institucionales, pero que no son controlados por los editores comerciales" (Schöpfel, 2010b). Las características actuales de este concepto están muy determinadas por la web 2.0, entre otras cosas, porque gran parte de esta producción está depositada en repositorios OAI, wikis, blogs, etc. y su producción es rápida e inmediata en muchos casos. Por tanto, no sería descabellado preguntarse si toda la web 2.0 es "gris", aunque nosotros preferimos pensar que ese concepto ya no existe. Pujol a este respecto y ya en 1995, vaticinaba cambios: "cada vez es más frecuente la producción de documentos en forma de literatura gris al ser más barata, más rápida y más sencilla de confeccionar. Por otro lado, el desarrollo de ediciones de documentos electrónicos, accesibles por medio de redes de comunicaciones, está incrementando el número de publicaciones que caen en este área gris (...) Dentro de unas décadas el concepto de literatura gris puede haber cambiado completamente".

Según Grey Net International, la asociación internacional organizadora de las conferencias internacionales sobre literatura gris, existe una amplia variedad de documentos que pueden acogerse a esta denominación (tabla 1).

En la tabla 1 llama la atención el amplio espectro de tipos documentales hoy en día partícipes de este concepto. De 


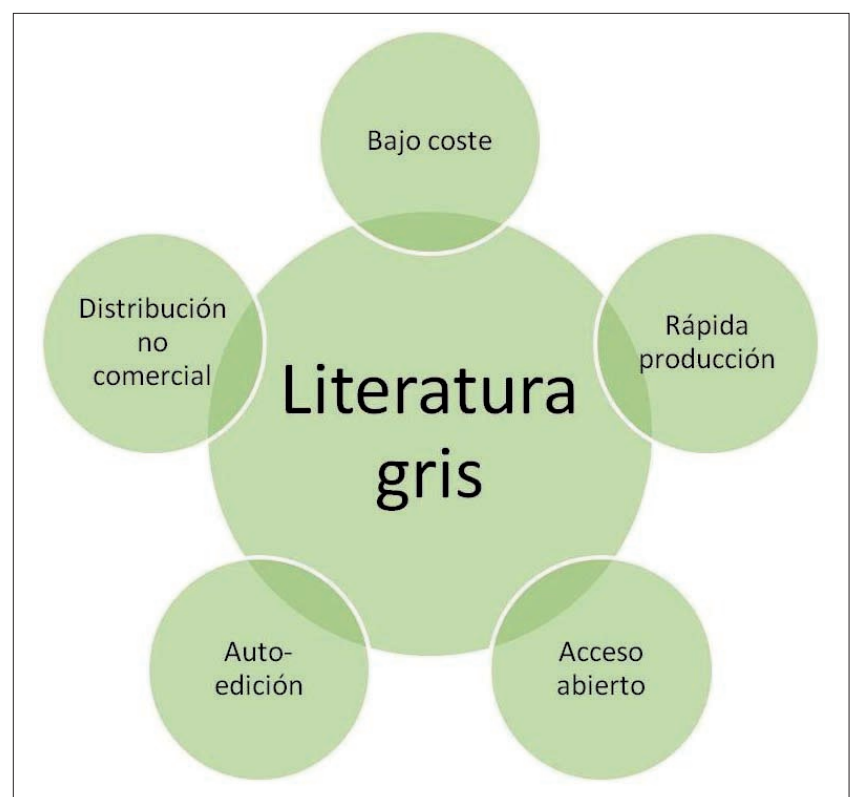

Figura 2. Características actuales de la literatura gris

hecho, prácticamente sólo están excluidas las monografías, porque hasta el artículo científico (su habitual 'partenaire' dentro de la comunicación científica) está incluido. Ciertamente hay que reconocer a los especialistas del tema su capacidad de adaptación y sus intentos de actualización, pero, al mismo tiempo se presenta la paradoja de que la aceptación de casi cualquier tipo documental y la nula distinción entre documento impreso y digital (distribuido preferentemente vía web y en muchos casos de forma libre y gratuita), entra en contradicción con la definición básica del concepto (acceso complicado y restringido), por no hablar de la localización del documento, mucho más dinámica en el momento actual. Por lo tanto, el hecho diferencial básico de la literatura gris -presente en la literatura científica y académica casi hasta el momento actual- no se justifica como tal en este momento. Si quisiéramos seguir manteniendo esta definición se deberían encontrar otros motivos, éste ya no parece vigente.

\begin{tabular}{|l|l|}
\hline Actas de congreso & Guías \\
Acuerdos de intercambio & Informes de investigación \\
Anuarios & Informes técnicos \\
Artículos & Lecciones inaugurales \\
Bases de datos & Legislación \\
Bibliografías & Libros blancos \\
Blogs & Libros verdes \\
Boletines & Manuales \\
Catálogos & Materiales de cursos \\
Conferencias & Normas \\
Convocatoria de artículos (call & Patentes \\
for papers) & Posters \\
Directorios & Protocolos científicos \\
Documentos de trabajo & Revistas \\
Ensayos & Software \\
Entrevistas & Tesis doctorales \\
E-prints & Traducciones \\
Estadísticas & \\
Estudios de caso & \\
\hline
\end{tabular}

Tabla 1: Lista de documentos grises. Fuente: GreyNet International http://www.greynet.org/greysourceindex/documenttypes.html

\section{Ocaso de la literatura gris}

Berners-Lee (1990) hablaba de la Web como un sistema de acceso universal a la información. En realidad es un sistema de acceso y de edición universal. La simplificación en el proceso editorial que aportó la Web en su momento (y no digamos ahora en el contexto de la web 2.0) es la verdadera razón del ocaso del concepto de literatura gris. La dificultad en el acceso a ciertos documentos de difusión restringida entra en conflicto con el acceso universal, y lo cierto es que no se puede nadar contracorriente, hoy en día es complicado encontrar tipos documentales que no hayan mejorado sensiblemente sus sistemas de publicación y de difusión (Pujol, 1995).

\section{El concepto 'literatura gris' está deter- minado por la tecnología web 2.0, ya que gran parte de esta producción está depositada en repositorios OAI, wikis, blogs, etc.}

El caso de las tesis doctorales es uno de los ejemplos más paradigmáticos de este proceso de cambio. Si bien siguen elaborándose de forma impresa (con copia electrónica generalmente), y suelen depositarse en las bibliotecas de las universidades donde se defienden, el clásico acceso limitado a estos documentos ha ido ampliándose por varias vías, bien a modo de publicación de la copia digital de la tesis dentro del repositorio institucional de la universidad o bien por medio de la edición de una copia de la misma en un repositorio especializado de tesis doctorales (generalmente multidisciplinares, como es el caso de TDR (Tesis doctorales en red), figura 3, o DART-Europe (portal europeo para el acceso abierto a tesis electrónicas). Si bien sigue siendo alto el número de tesis de acceso restringido, parece que se tiende a invertir esta situación.

http://www.tesisenred.net

http://www.dart-europe.eu

La difusión de las actas de congresos o conferencias ha aumentado exponencialmente desde la aparición de la Web. Si bien se suele mantener la tradicional edición impresa o en cd-rom de los congresos y jornadas que se entrega a los participantes, no es raro que los propios organizadores publiquen copias digitales en sus sitios web y que permitan que los participantes puedan publicar otras copias en sus propios sitios institucionales o en servidores eprints. Ejemplo del impacto de la web 2.0 sobre estos documentos es el cada vez mayor número de congresos y conferencias que emplean para la gestión y difusión de los contenidos el sistema Open conference systems (basado en la aplicación más famosa OJS para las publicaciones periódicas, solución de código abierto del proyecto Public Knowledge Project (PKP) de Canadá). Estos tipos documentales que han aumentado notablemente su acceso y difusión, no pueden considerarse hoy en día como documentos de alcance restringido.

Las normas constituyen un tipo tradicional de documento de carácter técnico y especializado, que habitualmente está infravalorado, pero han visto aumentada su difusión por la vía 


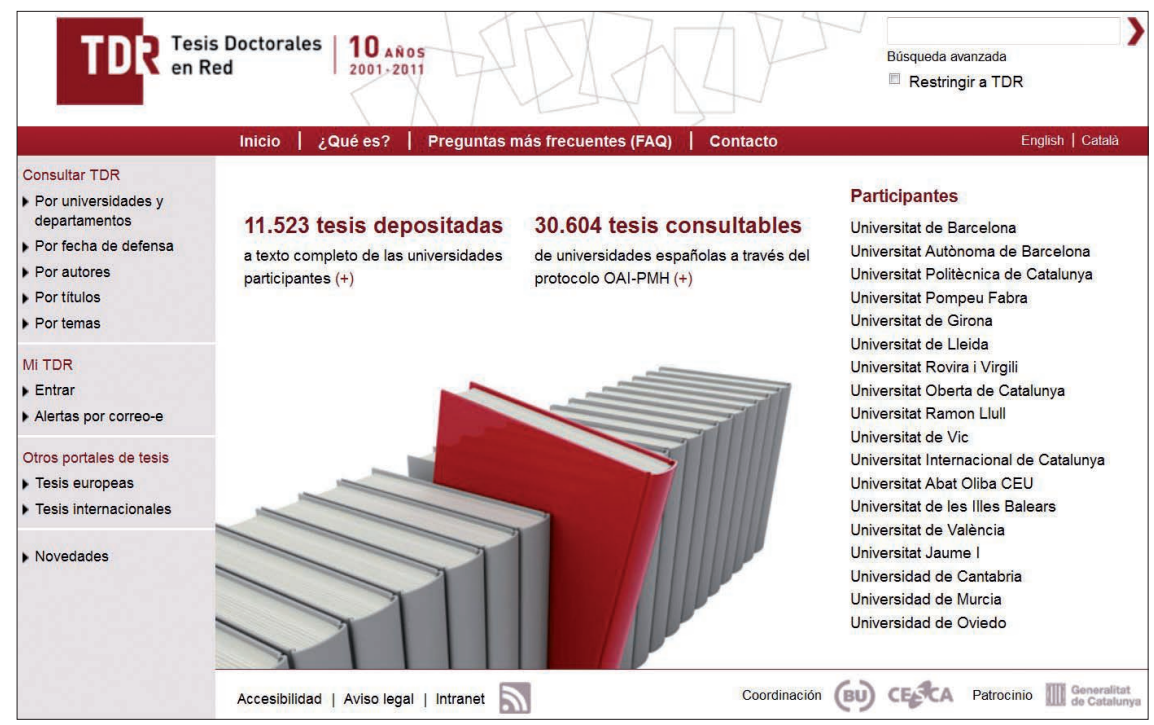

Figura 3. http://www.tesisenred.net

del portal web y la suscripción por medio de la sindicación de contenidos RSS. Si bien sigue siendo un documento cuya difusión no es muy extendida, ya que el número de usuarios potenciales es reducido y de carácter especializado, no cabe hablar de restricción y especialización en su acceso, más allá del coste de adquisición del documento mediante descarga de la Web de alguna de las organizaciones productoras (como pueden ser IEEE a nivel internacional o Aenor-UNE en el caso español).

Algo parecido sucede con las patentes y marcas. En este caso, el cambio es aún de mayor consideración, porque, sin renunciar a la especialización de este tipo documental, el número potencial de usuarios es mayor que en el caso de las normas y, en virtud de las características de estos documentos, la producción de bases de datos (tanto de consulta gratuita como de pago) es mucho mayor. La sindicación de contenidos vía RSS permite a los usuarios estar debidamente actualizados de las novedades que se produzcan en sus campos de interés. Al igual que ocurre con las normas, no cabe hablar de "documentos grises" porque sería confundir el carácter especializado de los documentos y de la comunidad de usuarios interesada con el acceso restringido.

\section{Los textos empleados en actividades do- centes corren peligro de quedarse obso- letos y desfasados}

Estos tipos documentales son sólo botones de muestra de una situación de cambio más global, porque ¿cabe hablar de un boletín o de un catálogo como documento de difícil acceso y difusión?, ¿puede decirse lo mismo de un blog, cuya vocación es sin duda alguna llegar al máximo número de usuarios posible? y ¿de verdad es tan difícil localizar la información de un call for papers para revistas o congresos científicos? (todos estos tipos documentales están en la tabla de Greynet). En este momento, la justificación de la existencia de esta denominación debería apoyarse en otros criterios, alejados ya del acceso y de la difusión del documento. Banks (2006) menciona que la tradicional dificultad en localizar y acceder a esos documentos es un criterio que debe dejar de usarse en breve. De hecho, si hoy en día aún persiste una escasa difusión de un documento, se debe, en la mayor parte de los casos a la intencionalidad de su productor de mantenerlo "semipublicado", bien porque se ha creado para un ámbito restringido y con una funcionalidad concreta, que no interesa difundir bien sea por seguridad (documentos oficiales que los gobiernos no pueden difundir, documentos de estudio de mercado de una empresa que evidentemente son de carácter reservado, etc.) o por mediocridad de los mismos (artículos, tesis o informes científicos no tan originales como debían de ser sin las debidas citas y referencias, informes de proyectos de escasa originalidad e incluso sin un debido tratamiento de datos y contraste de resultados, etc.).

Hoy resulta complicado hablar de documentos de alcance restringido y de difícil localización

\section{Discusión sobre la tradicional evaluación de la calidad de los trabajos}

Una vez perdida la batalla de los tipos documentales específicos de la literatura gris, algunos autores han intentado mantener en el tiempo el carácter especializado por medio de la discusión alrededor de dos matices: el control de la calidad de la producción científica (Tillet; Newbold, 2006) y el impacto de estos trabajos en la comunidad científica (Schöpfel, 2010a). Esta tendencia de pensamiento, si bien no de forma muy radical, intenta asentar la idea de equiparar literatura no sometida a un riguroso control bibliográfico (no distribuida por tanto por los medios tradicionales) a una menor calidad de sus contenidos, que redundará, sin duda alguna, en un menor impacto del contenido de la publicación, intentando así englobarla en una segunda categoría de la literatura científica, la "gris".

En este punto, sí que parecen estos autores haberse quedado aislados en una ínsula muy al margen de la evolución de la comunidad científica. Mostrar ahora reticencias sobre la calidad del contenido de estos documentos no es más que un vano intento de querer mantener unas posiciones que se tambalean desde hace tiempo. Más cuando es en algunas áreas científicas, especialmente en el campo de las matemáticas o la física donde encontramos el mejor ejemplo posible: la publicación de los resultados del Proyecto Opera sobre la velocidad de los neutrinos en arXiv.org y la tremenda discusión científica suscitada a su alrededor. Se confirma así que los repositorios digitales incluyen algunos de los más importantes preprints especializados y valorados por la comunidad científica, siendo a veces sitios obligados de pre-publicación 
del contenido de forma previa a su posible inclusión en una revista de impacto. Igual de absurdo sería rebajar la calidad de un blog (como puede ser el famoso Useit de Jakob Nielsen) por el mero hecho del soporte de difusión de la información. Introducir hoy en día dudas sobre el valor de una publicación, intentando hacer valer el peso del control bibliográfico como herramienta medidora de dicha calidad, resulta paradójico y anti natura (Banks, 2006).

No se trata ya de defender que la publicación en revistas científicas en la Web en acceso abierto posee la misma calidad que la realizada en las tradicionales revistas científicas de acceso restringido (bien en papel o en digital), para eso están los índices de impacto donde año tras año se comprueba el progresivo y paulatino éxito de las revistas científicas en acceso abierto. Esto serviría para desmontar el segundo argumento negativo citado anteriormente. De lo que se trata ahora es de verificar si este entorno está contribuyendo a una mayor difusión de los documentos, tanto los tradicionalmente calificados como grises como los convencionales tal como afirman Cool (2007), Smith (2008) y Peters, (2009). El desarrollo de las herramientas web 2.0, como los blogs, wikis, redes sociales y repositorios OAI han mejorado extraordinariamente la localización de documentos que tradicionalmente consideraríamos como "grises", creándose valiosas fuentes de información en áreas especializadas como, por ejemplo, son Grey literature report en medicina o repositorios institucionales como es el caso de OpenSigle. Algunos autores plantean la necesidad de conservar el contenido generado por estas herramientas 2.0 (Banks, 2009) especialmente en aquellos casos donde se evidencien signos de calidad y relevancia en el ámbito académico-científico, e incluso algunos de ellos llegan a hablar (no podía ser de otro modo vista la tendencia actual en otros campos) de una nueva literatura gris 2.0. Si bien estamos de acuerdo en el reconocimiento de esa importancia, no consideramos oportuna esta definición, no ya por el tan traído y tan llevado (a veces en exceso) calificativo " 2.0 ", sino más bien porque creemos que ya no tiene razón de ser la propia idea, independientemente de la "versión" de la Web en la que nos encontremos.

Pretender valorar de alguna manera el impacto que puede tener lo publicado en el entorno de la web 2.0 es un campo emergente de trabajo e investigación en estos momentos, donde ya se comienzan a introducir cambios importantes en la evaluación de lo publicado que deben aún ir desarrollándose completamente pero que demuestran el hecho imparable de todo este proceso. Si, tradicionalmente, la calidad de una aportación científica se valora por medio del factor de impacto de la revista donde se publique (medida que parte del total de citas recibidas), aquellos artículos publicados en revistas no incluidas en estos índices o rankings resultan de difícil valoración. En el contexto de la web 2.0 ya no es correcto restringir la cita al artículo científico, sino que deben tomarse en consideración otro tipo de documentos (un post sin ir más lejos o una página personal donde, por ejemplo, el inventor de la Web recoge una copia de la propuesta original). En este nuevo contexto el número de citas puede aumentar considerablemente, siendo muchas de ellas copias literales de la original. Esto propicia que ahora se está comenzando a hablar de "citas positivas recibidas", esto es, contabilizar la cita sólo cuando la misma enriquece a las nuevas argumentaciones que se exponen, excluyendo aquellas que son un simple repaso del estado de la cuestión: "en el pasado, los indicadores de impacto se limitaban a las citas y revistas. Hoy en día, las mediciones del uso ofrecen nuevas oportunidades para medir el impacto de gran escala de los recursos digitales, estos indicadores proporcionarán información adicional sobre la calidad y la popularidad para el usuario final" (Schöpfel, 2010a).

\section{De persistir la tendencia de embargar en exceso la difusión de sus contenidos, los artículos científicos están en peligro de pasar desapercibidos}

Pero el cambio no acaba ahí. No se trata ya de adaptar las fórmulas tradicionalmente empleadas para medir ese factor de impacto, ahora se trata de -emulando en cierto modo el "Renacimiento" de O'Reilly- de recrear esas fórmulas. Así, en Mendeley.com (2009) están trabajando en métodos alternativos para valorar el impacto de lo publicado en cualquier sitio de la web 2.0, haciendo posible que:

- ahora podamos medir el impacto de un artículo individualmente en términos de número de lectores (cuántas descargas, el tiempo medio dedicado a la lectura, la frecuencia con que el artículo es compartido, etc.);

- medida en tiempo real: no hay que esperar dos años o más antes de ver con qué frecuencia un artículo se cita para determinar su valor;

- se nivela el terreno de juego para todas las fuentes de información. De las estadísticas de lectores, cada artículo puede ahora ser evaluado individualmente por sus propios méritos y no por la revista o blog que lo acoge.

Como se ve, se están introduciendo cambios a la hora de generar estadísticas (y los cálculos tradicionales de la comunidad científica se acercan un poco a los parámetros empleados habitualmente en la comunidad web 2.0), pero esto no acaba ahí: también se pretende fomentar la interacción de repositorios digitales con redes sociales que permitirá ampliar el número de expertos en tareas de revisión y valoración, contribuyendo a aumentar ineludiblemente su difusión e impacto (Melero, 2005). La web 2.0 está provocando una nueva paradoja, las revistas que retrasan su acceso abierto a los contenidos son las que están restringiendo su difusión. De persistir la tendencia de embargar en exceso la difusión de sus contenidos, los artículos científicos -que son los que vehiculan la comunicación científica desde hace casi tres siglos publicados en revistas científicas- están en peligro de comenzar a pasar inadvertidos, al menos una parte considerable de ellos, como si fueran "grises" en el sentido clásico del término.

El asunto pendiente estaría en crear nuevos métodos de análisis y evaluación científica de esta literatura que permita a instituciones científicas e investigadores conocer con certeza el verdadero estado del arte en sus disciplinas y quiénes son realmente los más productivos desde un punto de vista de contribución científica. Cada tipo documental tiene unas 
características intrínsecas: un artículo científico es sometido a rondas de evaluación, una tesis doctoral es revisada por el director y un tribunal especializado, una comunicación a congreso también suele ser evaluada con anterioridad, etc. Por tanto, puede ser que no exista un método universal, pero en todo caso, debe quedar claro que todos coinciden en una cosa: la voluntad de sus autores en que el contenido de su aportación sea difundido, y a cuantos más usuarios mejor.

El mundo, en la época 2.0, ya no parece tan gris.

\section{Conclusiones}

La bibliografía actual en torno a literatura gris, sobre todo en castellano, sigue empleando definiciones clásicas que nada tienen que ver con la realidad. Hay que proceder a una revisión de estos textos en la línea que hemos pretendido transmitir en este trabajo: este concepto ha perdido su razón de ser en el contexto de la web 2.0 y no tiene sentido seguir hablando del mismo, entre otras cosas porque de persistir en esta discusión, se podría llegar a concluir que todo lo publicado en este nuevo contexto forma parte de la literatura gris, y esto tampoco es cierto.

De forma adicional, creemos llegado el momento de llamar la atención sobre nuevas perspectivas de trabajo e investigación en la valoración de la producción científica, especialmente de toda aquella que no se desarrolla por los cauces habitualmente recogidos por los índices de impacto, que ahora está siendo prácticamente ignorada.

Algunos autores hablan de una nueva 'literatura gris 2.0'. Creemos que esa idea ya no tiene razón de ser

\section{Nota}

1. http://es.wikipedia.org/wiki/WebQuest

\section{Referencias}

Banks, Marcus A. "Towards a continuum of scholarship: the eventual collapse of the distinction between grey and nongrey literature?" En: GL7 Conference proceedings. TextRelease, 2006.

http://eprints.rclis.org/handle/10760/7287

Banks, Marcus A. "Blog posts and tweets: the next frontier for grey literature". En: Farace, Dominic; Schopfel, Joachim. Grey literature in library and information studies. De Gruyter, 2009.

http://eprints.rclis.org/handle/10760/15411

Berners-Lee, Tim. WorldWideWeb: Proposal for a hypertext project. W3C, 1990.

http://www.w3.org/Proposal.html

Carrizo-Sainero, Gloria. La información en ciencias sociales. Gijón: Trea, 2000, 284 pp. ISBN 8495178850

Cool, Heidi. "Web 2.0 \& social networking can enhance 'findability'”. Web development blog, 2007.

http://blog.case.edu/webdev/2007/07/23/social.html
Cordón-García, José-Antonio. Manual de búsqueda documental y práctica bibliográfica. Madrid: Pirámide, 1999. 238 pp. ISBN 9788436812022

Currás, Emilia. "Sistema experto hipermedia para el reconocimiento, indización y recuperación de literatura gris". Scire, 1998 , v. 4, n. 1, pp. 117-130.

http://ibersid.eu/ojs/index.php/scire/article/view/1091/ 1073

De-Torres-Ramírez, Isabel. Las fuentes de información: estudios teórico-prácticos. Madrid: Síntesis, 1998. ISBN 8477384606

Daugherty, Dale. http://en.wikipedia.org/wiki/Dale_Dougherty

http://www.oreillynet.com/pub/au/26

Martín-Vega, Arturo. Fuentes de información general. Gijón: Trea, 1995. ISBN 8489427291

Melero, Remedios. "Acceso abierto a las publicaciones científicas: definición, recursos, copyright e impacto". El profesional de la información, 2005, v. 15, n. 4. pp. 256-266. http://eprints.rclis.org/bitstream/10760/6571/1/EPI-rmele ro.pdf

mendeley.com. Ologeez founder joins Mendeley / Changing the journal impact factor, 2009.

http://www.mendeley.com/blog/academic-features/olo geez-founder-joins-mendeley

Ramos-de-Carvalho, Elizabet-Maria. "La literatura gris y su contribución a la sociedad del conocimiento". 67th IFLA Council and general conf. Boston, August 16-25, 2001. http://ifla.queenslibrary.org/iv/ifla67/papers/090-173s. $p d f$

Peters, Isabella. Folksonomies: indexing and retrieval in web 2.0. Berlín: Walter de Gruyter, 2009. 443 pp. ISBN 9783598251795

Pujol, Rosa. "La literatura gris en expansión". Information world en español, 1995, n. 32.

http://www.elprofesionaldelainformacion.com/conteni dos/1995/marzo/la_literatura_gris_en_expansin.html

Schöpfel, Joachim. "Access to European grey literature". En: Grey literature repositories, 2010a, pp. 20-33.

http://archivesic.ccsd.cnrs.fr/docs/00/59/77/98/PDF/Schop fel_4.0.pdf

Schöpfel, Joachim. "Towards a Prague definition of grey literature". En: Proceedings - Twelfth intl conf on grey literature. Transparency in grey literature. Grey tech approaches to high tech issues. Prague 6-7 Dec. 2010.

http://archivesic.ccsd.cnrs.fr/docs/00/58/15/70/PDF/GL_ 12_Schopfel_v5.2.pdf

Smith, Gene. Tagging: people-powered metadata for the social web. Berkeley, CA: New Riders, 2008, 208 pp. ISBN 9780321529176

Tillet, Samantha; Newbold, Elizabeth. "Grey literature at the British Library: revealing a hidden resource". Interlending \& document supply, 2006, v. 34, n. 2, pp. 70-73. http://dx.doi.org/10.1108/02641610610669769 